\title{
AN AXISYMMETRIC BENDING AND SHEAR STRESS ANALYSIS OF OF FUNCTIONALLY GRADED CIRCULAR PLATE BASED ON UNCONSTRAINED THIRD ORDER SHEAR DEFORMATION THEORY VIA DIFFERENTIAL QUADRATURE METHOD
}

\author{
HamadM.H ${ }^{1}$, F. Tarlochan ${ }^{2}$ \\ Center for Innovation and Design, College of Engineering, UniversitiTenagaNasional, 43009 Selangor
}

\begin{abstract}
In this study, based on the unconstrained third order shear deformation theory (UTSDT), numerical analysis of an axisymmetric bending and stresses of circular plate are investigated. The material properties are considerd to graded through the thickness of the verticlecoordinate, and follow a simple power of volume fraction of the constituents.governing equations are derived and DQM is used as an efficient numerical method for solving the differential equations. Two types of boundary conditions under the influence of the bending and body force are studied. The validation of the results is done by a comparison with another study, which available in the literature and found good agreement between two studies.
\end{abstract}

Index Terms:bending, shearstress, circularplate,UTSDT,GDQM.

\section{INTRODUCTION}

Thick and thin Circular disk in structured componentsplays a major role in engineering applications related to this area is the static analysis thetypes of plates which arenotably crucial in their design ranging from automotive railway brake systems to disks which constitute vital components particularly in turbo machines. Functionally,graded materials (FGMs) were first introduced in 1989 [1] whereby a number of researchers, because interested to study them .

In the past decade, many of the studies which carried out on the FGMs disks concentrated on the conventional plate and the first order shear deformation theories. The conventional plate theory (CPT) furnishes accurate and reliableanalysis for this plate. As the disk thickness increases CPT over predicts stresses response, because the transverse shear deformation and rotary inertia effects are neglected .So there a number of shear deformation theories used to analyze moderately thick plate, first order theory and third order theory were developed to incorporate the shear deformation effects, in the first order shear deformation theory (FSDT), the constant shear stress condition through thicknesses violates the statically condition of zero shear stress at the free surface. So its need for shear correction factor to modify the shear forces .The third order shear deformation theory (TSDT )predicts parabolic variation of shear stress through the thickness. Although the use of higher order plate theory leads to more an accurate prediction of the global response quantities such as shear forces, deflections strain and stresses, it requires much computation effort . Furthermore the use of the (TSDT) by Reddy is constrained, because it considers the shear stress vanishes on the top and bottom surfaces of the plate, but this limitation is solved by the unconstrainedthird order shear deformation theory (TSDT) by Leuny [2].

In past decades several studies published on the static analysis of FGMs circular disks Reddy et al. [3] Study relates to ax symmetric bending of functionally graded circular and annular plates whereby the first order shear deformation plate theory was used. Ma and Wang [4] analyzed further by discussing the relationship between axisymmetric bending and buckling solutions of FGM circular plates. Third-order plate theory and classical plate theory were demonstrated and discussed in detail in their study. In addition, asymmetric flexural vibration and an additional stability analysis of FGM circular plates was included in thermal environment by using finite element techniquespresented by Prakash and Ganapathi [5]. Also, Three-dimensional free vibration of functionally graded annular plates whereby boundary conditions were different using a Chebyshev-Ritz method was also studied by Dong [6]. Malekzadeh et al. [7] Also showed how in thermal environment in-plane free vibration analysis of FGM thin-tomoderately thick deep circular arches. Third-order shear deformation theory was used by Saidi et al. [8] To analyze axisymmetric bending and buckling of thick functionally graded circular plates. Subsequently, fourth-order shear deformation theory was researched by Sahraee and saidi [9] to study axisymmetric bending of thick functionally graded 
circular plates. Besides this, Sepahi et al. [10] Analyzed the effects of big deflection of thermo-mechanical loaded annular FGM plates on nonlinear elastic foundation using the differential quadrature method. Geometrically nonlinear postbuckling of an imperfect circular FGM plate was studied by Li et al. [11] who found both mechanical load and transverse non-uniform temperature rise. A study conducted by Malekzadeh et al. [12] resulted in three-dimensional free vibration of thick functionally graded annular plate in thermal environment by differential quadrature technique. An investigation of nonlinear analysis of functionally graded circular plates was conducted by Nosier and Fallah [13] It was related to asymmetric transverse loading, according to the first-order shear deformation plate theory based on von Karman non-linearity. In addition, Sburlati and Bardella [14] studied three-dimensional elasticity solution of functionally graded thick circular plates. Correspondingly, Golmakani and Kadkhodayan [15] studied axisymmetric nonlinear bending analysis of annular functionally graded plate. The study used third-order shear deformation theory. A precise closed form answer for free vibration of circular and annular moderately thick functionally graded plates of first-order shear deformation theory was studied by Hosseini-Hashemi et al. [16] Nie and Zhong's study [17] was on frequency analysis of multi-directional functionally graded annular plates using state space differential quadrature method. It was based on the three-dimensional theory of elasticity. It must be noted that direct displacement method was conducted by Yan et al. [18] with the aim to represent the axisymmetric bending of FG circular plates under transverse loads that were arbitrary. Another theory using Mindlin's plate theory about free vibration was investigated by Ebrahimi et al [19]. This study was concerned about moderately thick shear deformable annular functionally graded by Pilate. The effects of coupling between in-plane and out-of-plane vibrating modes of smart functionally graded circular/annular plates was examined by Hashemi et al. [20]. Nonlinear bending and post-buckling of a functionally graded circular plate was examined by Ma and Wang [21] whereby the conditions were mechanical and thermal loading. Bayat et al [22 ]used first-order shear deformation theory to study the thermal elastic response of rotating disk with small and large deflections, and presented the results with analytical solutions.

Viola et al. [23] Used a2D unconstrained third order shear deformation theory (UTSDT) in static analysis of moderately thick functionally graded cylindrical shells subjected to mechanical loadings. Also Viola et al. [24] Employed (UTSDT) for analyzing thedynamicbehaviour of completely doubly-curved laminated shells and panels .

It is clear from the above literature most of the studies which carried out on the static analysis of the circular disks based on the first order shear deformation theory and few of them based on the third order deformation theory which is solved analytically for limited boundary conditions. On the other hand the numerical technique presenting static analysis for the circular disks based on high unconstrained third order shear deformation theory is quite poor.As well as, the use of the shear function model which is used by [24] can by applied to the displacement field of the circular disk .

In the present study,unconstrainedthird order shear deformation theory is used for axisymmetric static analysis of functionally graded clamped and rolatingcirculer plate .The circular plate is subjected to two types of loading, bending and body force. The mechanical properties are assumed to be graded in the thickness direction according to a simple power law distribution in terms of the volume fraction of the constituent . By the principle of minimum total energy, the governing equations of equilibrium are obtained according to the unconstrained third order shear deformation theory. By employing the differential quadrature method as a simple but accurate and fast convergent method to discretizethe equilibrium equations and to implement the boundary conditions. The effects of body force parameters ,the material constant, and the geometric parameters of circular plate on the stresses and deflection response are is studied in detail.

\section{PROBLEM STATEMENT}

Consider a FG circular disk with thickness( h ) and radius ( a ), axisymmetric with respect to the $\mathrm{z}$-axis as shown in fig. 1 and it is subjected to uniform transverse pressure in case of clamped condition, whilein case of roller support condition, it subjected to both uniform pressure and body force

The mid-plane of the plate refers to the cylindrical coordinate system $^{(r, \theta, z)}$ in the radial ,circumferential and axial directions respectively.

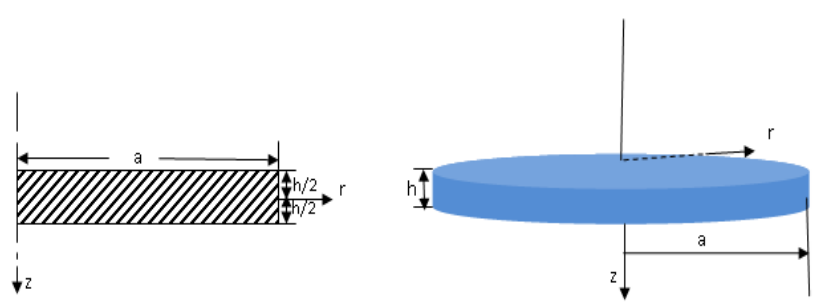

Figure 1:FG circular disk with thickness (h) and radius (a)

\section{1- Mechanical Properties OfFGCircular Plate}

\section{Types}

Typically FGMs are made of a mixture of the two constituents. In this research it is assumed that the FGMs are made of a mixture of ceramic and metal constituents.the material properties of the FG plate vary continuously and smoothly in thickness direction $\mathrm{z}$ and are functions of volume fraction of constituent materials 


$$
P(z)=(P m-P c) V m+P c_{(1)}
$$

Where:

$P(z)$ :material property at location z through thickness.m and c denotes the metallic and ceramic constituents respictivlly .

Vm :volume fraction of metal

$$
V_{m}=\left(\frac{h-2 z}{2 h}\right)^{p}
$$

Where:

z: thickness coordinates $(-h / 2 \leq z \leq h / 2)$

$\mathrm{p}:$ material constant.

As the material constant is equl to zero $(p=0)$, or equal to infinity $(p=\infty)$, the homogeneous isotropic material is obtained as a specializ case of functioaly graded material . in fact , from equation (2) it possible to obtaian :

$$
\begin{aligned}
& p=0 \rightarrow V_{m}=1, V_{c}=0 \rightarrow p(z)=p_{m} \\
& p=\infty \rightarrow V_{m}=0, V_{c}=1 \rightarrow p(z)=p_{c}
\end{aligned}
$$

Fig.2 show that material profile through the FG plate for various of $\mathrm{p}$.

According to relution (1), the elastic modulur E and density $\rho$ to be varied according to the above equation . andpoisson's

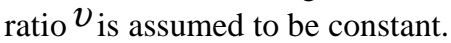

$$
\begin{gathered}
E(z)=\left(E_{m}-E_{C}\right)+V_{m}+E_{c}(3) \\
\rho(z)=\left(\rho_{m}-\rho_{C}\right) V_{m}+\rho_{c}(4)
\end{gathered}
$$

\section{GOVERING EQUATIONS}

Based on the unconstrained thired-order shear deformation theory (UTSDT), displacement field in the cylindrical coordinate system can be written as :

$$
\begin{gathered}
U(r, z)=u(r)+f(z) \phi_{1}(r)+g(z) \phi_{2}(r)(5) \\
w(r, z)=w(r)(6)
\end{gathered}
$$

Where : $\mathrm{u}, \mathrm{w}$ are the displacements of points on the middle plane $(\mathrm{z}=0)$ in the radial and vertical direction respictivly. $\phi_{1}$ :smalltransrverse normal rotation about the $\theta_{\text {-axises }}$

$\phi_{2}$ : smalltransrverse normal higher order rotation about $\theta_{\text {_ }}$ axises

$$
f(z), g(z) \text { are shear functions. }
$$

From the previous[23], the displacement field has been improved by taking into consideration shear functions along the thinckess . indeed ,the model for the shear function in this study has taken from previous work[24].

Strain- displacement relations:

$$
\begin{gathered}
\varepsilon_{r r}=\frac{d u}{d r}+\left(z-\alpha z^{3}\right) \frac{d \phi_{1}}{d r}-\alpha z^{3} \frac{d \phi_{2}}{d r} \\
\varepsilon_{\theta \theta}=\frac{1}{r} u+\left(z-\alpha z^{3}\right) \frac{1}{r} \phi_{1}-\alpha z^{3} \frac{1}{r} \phi_{2} \\
\gamma_{r z}=\left(1-3 \alpha z^{2}\right) \phi_{1}-3 \alpha z^{2} \phi_{2}+\frac{d w}{d r} \\
\gamma_{r \theta}=0, \gamma_{\theta z}=0, \varepsilon_{z}=0
\end{gathered}
$$

Stress-strain relations

$$
\left\{\begin{array}{c}
\sigma_{\mathrm{r}} \\
\sigma_{\theta} \\
\tau_{\mathrm{rz}}
\end{array}\right\}=\left[\begin{array}{ccc}
Q_{11} & Q_{12} & 0 \\
Q_{12} & Q_{22} & 0 \\
0 & 0 & Q_{66}
\end{array}\right]\left\{\begin{array}{c}
\varepsilon_{\mathrm{r}} \\
\varepsilon_{\theta} \\
\gamma_{\mathrm{rz}}
\end{array}\right\}
$$

Where :

$$
\begin{gathered}
Q_{11}=Q_{22}=\frac{E(z)}{2\left(1+v^{2}\right)}, Q_{12}=v Q_{11} \\
Q_{66}=\frac{\mathrm{E}(\mathrm{z})}{2(1+v)}
\end{gathered}
$$

The total potential energy of circular plate:

$$
\Pi=\mathrm{U}+\mathrm{V}_{\mathrm{f}}(9)
$$




$$
\begin{aligned}
& V_{f}=-2 \pi \int_{0}^{a}\left(\rho_{1} r^{2} \omega^{2} \delta u+\rho_{2} r^{2} \omega^{2} \delta \phi_{1}\right. \\
& \left.-\alpha \rho_{3} r^{2} \omega^{2} \delta \phi_{1}-\alpha \rho_{3} r^{2} w^{2} \delta \phi_{2}+r q \delta w\right) d r
\end{aligned}
$$

\section{Where :}

$\Pi, U, V_{f}$ are the total potential energy, strain energy and the potential energy of the body force and pressure load respectively.

By the principle of minimum total energy $\delta \Pi=0$

$$
\int_{0}^{a}\left\{\begin{array}{l}
\left(\begin{array}{l}
\left.-\frac{d}{d r}\left(r N_{r}\right)+N_{\theta}-\rho_{1} r^{2} \omega^{2}\right) \delta u+ \\
\left(-\frac{d}{d r}\left(r M_{r}\right)+\alpha \frac{d}{d r}\left(r p_{r}\right)+M_{\theta}-\alpha p_{\theta}+r \phi_{r}-\right. \\
\left(\alpha \rho_{r}+\right. \\
\left(\alpha \frac{d}{d r}\left(r p_{r}\right)-\alpha p_{\theta-} 3 \alpha r R_{r}+\alpha \rho_{3} r^{2} \omega^{2}\right) \delta \phi_{1}+ \\
\left(\frac{d}{d r}\left(r \phi_{r}\right)+r q_{r}\right) \delta w
\end{array}\right.
\end{array}\right\} d r=0
$$

Where:

$\mathrm{N}_{\mathrm{r}}, \mathrm{N}_{\theta}:$ Stress resultants

$\mathrm{M}_{\mathrm{r}}, \mathrm{M}_{\theta}:$ Stress couples

$p_{r}, p_{\theta}:$ higher order stress couples

$\phi_{r}$ :transverse shear resultant

$R_{r}$ : higher order shear resultant

$\rho_{1,} \rho_{2}, \rho_{3}$ : constant proportional to the mean ,first and third moment of the density along the thickness.

$$
\begin{aligned}
\left(N_{r}, M_{r}, P_{r}\right) & =\int_{-h / 2}^{h / 2} \sigma_{r}\left(1, z, z^{3}\right) d z \\
\left(N_{\theta}, M_{\theta}, P_{\theta}\right) & =\int_{-h / 2}^{h / 2} \sigma_{\theta}\left(1, z, z^{3}\right) d z \\
\left(\phi_{r}, R_{r}\right) & =\int_{-h / 2}^{h / 2} \tau_{r z}\left(1, z^{2}\right) d z
\end{aligned}
$$

$$
\left(\rho_{1}, \rho_{2}, \rho_{3}\right)=\int_{-h / 2}^{h / 2} \rho(z)\left(1, z, z^{3}\right) d z
$$

From eqs. (7),(8) and (16), one can obtain the following relations:

$$
\begin{aligned}
& N_{r}=A_{11}\left(\frac{d u}{d r}+v \frac{1}{r} u\right)+ \\
& \left(B_{11}-\alpha E_{11}\right)\left(\frac{d \phi_{1}}{d r}+v \frac{1}{r} \phi_{1}\right)-\alpha E_{11}\left(\frac{d \phi_{2}}{d r}+v \frac{1}{r} \phi_{2}\right)
\end{aligned}
$$

$$
\begin{aligned}
& N_{\theta}=A_{11}\left(v \frac{d u}{d r}+\frac{1}{r} u\right) \\
& +\left(B_{11}-\alpha E_{11}\right)\left(v \frac{d \phi_{1}}{d r}+\frac{1}{r} \phi_{1}\right)-\alpha E_{11}\left(v \frac{d \phi_{2}}{d r}+\frac{1}{r} \phi_{2}\right) \\
& M_{r}=B_{11}\left(\frac{d u}{d r}+v \frac{1}{r} u\right) \\
& +\left(D_{11}-\alpha F_{11}\right)\left(\frac{d \phi_{1}}{d r}+v \frac{1}{r} \phi_{1}\right)-\alpha F_{11}\left(\frac{d \phi_{2}}{d r}+v \frac{1}{r} \phi_{2}\right)
\end{aligned}
$$$$
M_{\theta}=B_{11}\left(v \frac{d u}{d r}+\frac{1}{r} u\right)
$$$$
+\left(D_{11}-\alpha F_{11}\right)\left(v \frac{d \phi_{1}}{d r}+\frac{1}{r} \phi_{1}\right)-\alpha F_{11}\left(v \frac{d \phi_{2}}{d r}+\frac{1}{r} \phi_{2}\right)
$$$$
P_{r}=E_{11}\left(\frac{d u}{d r}+v \frac{1}{r} u\right)+
$$$$
\left(F_{11}-\alpha H_{11}\right)\left(\frac{d \phi_{1}}{d r}+v \frac{1}{r} \phi_{1}\right)-\alpha H_{11}\left(\frac{d \phi_{2}}{d r}+v \frac{1}{r} \phi_{2}\right)
$$$$
Q_{r}=A_{44}\left(\phi_{1}+\frac{d w}{d r}\right)-3 \alpha D_{44}\left(\phi_{1}+\phi_{2}\right)
$$$$
R_{r}=D_{44}\left(\phi_{1}+\frac{d w}{d r}\right)-3 \alpha F_{44}\left(\phi_{1}+\phi_{2}\right)
$$

Where:

$A_{11}, B_{11}, D_{11}, E_{11}, F_{11}, H_{11}$ : are the circulaer disk stiffness coefficients 


$$
\begin{aligned}
& \left(A_{11}, B_{11}, D_{11}, E_{11}, H_{11}\right)= \\
& \int_{-h / 2}^{h / 2} \frac{E(z)}{\left(1-v^{2}\right)}\left(1, z, z^{2}, z^{3}, z^{4}, z^{6}\right) d z
\end{aligned}
$$

$$
\left(A_{44}, D_{44}, F_{44}\right)=\int_{-h / 2}^{h / 2} \frac{E(z)}{2(1-v)}\left(1, z, z^{2}, z^{4}\right) d z
$$

From equation (12), the equilibrium equations are $\delta u$ :

$$
\frac{d}{d r}\left(r N_{r}\right)+N_{\theta}-\rho_{1} r^{2} \omega^{2}=0
$$

$\delta \phi_{1}:$

$\frac{d}{d r}\left(r M_{r}\right)-\alpha \frac{d}{d r}\left(r P_{r}\right)-M_{\theta}+\alpha P_{\theta}-r Q_{r}+3 \alpha r R_{r}+$ $\left(\rho_{2}-\alpha \rho_{3}\right) r^{2} \omega^{2}=0$

$\delta \phi_{2}:$

$$
\alpha \frac{d}{d r}\left(r P_{r}\right)-\alpha P_{\theta}-3 \alpha r R_{r}+\alpha \rho_{3} r^{2} \omega^{2}=0
$$

$\delta w:$

$$
\frac{d}{d r}\left(r Q_{r}\right)+r q=0
$$

Equilibrium equations in terms of displacements:

$$
\begin{aligned}
& \left(A_{11} r\right) \frac{d^{2} u}{d r^{2}}+A_{11} \frac{d u}{d r}-A_{11} \frac{1}{r} u+\left(B_{11}-\alpha E_{11}\right) r \frac{d^{2} \phi_{1}}{d r^{2}}+ \\
& \left(B_{11}-\alpha E_{11}\right) \frac{d \phi_{1}}{d r}-\left(B_{11}-\alpha E_{11}\right)+ \\
& \frac{1}{r} \phi_{1}-\alpha E_{11} r \frac{d^{2} \phi_{2}}{d r^{2}}-\alpha E_{11} \frac{d \phi_{2}}{d r}+\alpha E_{11} \frac{1}{r} \phi_{2}+\rho_{1} r^{2} \omega^{2}=0
\end{aligned}
$$

$$
\begin{aligned}
& \left(B_{11}-\alpha E_{11}\right) r \frac{d^{2} u}{d r^{2}}+\left(B_{11}-\alpha E_{11}\right) \frac{d u}{d r}-\left(B_{11}-\alpha E_{11}\right) \frac{1}{r} u+ \\
& \left(D_{11}-2 \alpha F_{11}+\alpha^{2} H_{11}\right) \frac{d \phi_{1}}{d r} \\
& -\left[\left(D_{11}-2 \alpha F_{11}+\alpha^{2} H_{11}\right) \frac{1}{r}+\left(A_{44}-6 \alpha D_{44}+9 \alpha^{2} F_{44}\right) r\right] \phi_{1}- \\
& \left(\alpha F_{11}+\alpha^{2} H_{11}\right) r \frac{d^{2} \phi_{2}}{d r^{2}} \\
& -\left(\alpha F_{11}+\alpha^{2} H_{11}\right) \frac{d \phi_{2}}{d r}+\left[\left(\alpha F_{11}+\alpha^{2} H_{11}\right) \frac{1}{r}+\left(3 \alpha D_{44}+9 \alpha^{2} F_{44}\right) r\right] \phi_{2} \\
& -\left(A_{44}-3 \alpha D_{44}\right) \frac{d w}{d r}+\left(\rho_{2}-\rho_{3}\right) r^{2} \omega^{2}=0
\end{aligned}
$$

$$
\begin{aligned}
& E_{11} r \frac{d^{2} u}{d r^{2}}+E_{11} \frac{d u}{d r}-E_{11} \frac{1}{r} u\left(F_{11}+\alpha H_{11}\right) r \frac{d^{2} \phi_{1}}{d r^{2}} \\
& +\left(F_{11}+\alpha H_{11}\right) \frac{d \phi_{1}}{d r}-\left[\left(F_{11}+\alpha H_{11}\right) \frac{1}{r}+\left(3 D_{44}-9 \alpha F_{44}\right)\right] r \phi_{1} \\
& -\alpha H_{11} r \frac{d^{2} \phi_{1}}{d r^{2}}-\alpha H_{11} \frac{d \phi_{1}}{d r}+\left(\alpha H_{11} \frac{1}{r}+9 \alpha E_{44} r\right) \\
& \phi_{2}-3 D_{44} r \frac{d w}{d r}+\alpha \rho_{3} r^{2} \omega^{2}=0
\end{aligned}
$$

$$
\begin{aligned}
& \left(A_{44}-3 \alpha D_{44}\right) r \frac{d \phi_{1}}{d r}+\left(A_{44}-3 \alpha D_{44}\right) \phi_{1}- \\
& \left(3 \alpha D_{44}\right) r \frac{d \phi_{2}}{d r}-\left(3 \alpha D_{44}\right) \phi_{2}+A_{44} r \frac{d^{2} w}{d r^{2}}+ \\
& r q=0
\end{aligned}
$$

Using the following dimensionless parameters for simplicity .

$$
\begin{gathered}
R=\frac{r}{a}, W=\frac{w}{h}, U=\frac{u h}{a^{2}}, \varphi=\phi \quad \delta=\frac{h}{a}, \eta_{1}=\frac{A_{11} h^{6}}{H_{11}}, \\
\eta_{2}=\frac{B_{11} h^{5}}{H_{11}}, \quad \eta_{3}=\frac{D_{11} h^{4}}{H_{11}}, \\
\eta_{4}=\frac{E_{11} h^{3}}{H_{11}}, \eta_{5}=\frac{F_{11} h^{2}}{H_{11}}, \eta_{6}=\frac{A_{44} h^{6}}{H_{11}}, \eta_{7}=\frac{D_{44} h^{4}}{H_{11}}, \\
\eta_{8}=\frac{F_{44} h^{2}}{H_{11}}
\end{gathered}
$$


$Q:$ pressure parameter

$$
Q=\frac{h^{6} * q}{H_{11}}
$$

$\zeta:$ bodyforec parameters

$$
\begin{gathered}
\zeta_{1}=\frac{\rho_{1} h^{7} a \omega^{2}}{H_{11}} \\
\zeta_{2}=\frac{\left(\rho_{2}-\alpha \rho_{3}\right) h^{8} \omega^{2}}{\alpha H_{11}} \\
\zeta_{3}=\frac{4 \rho_{3} h^{4} \omega^{2}}{3 a H_{11}}
\end{gathered}
$$

the equations of motion in dimensionless form are:

$$
\begin{aligned}
& \eta_{1} \delta R \frac{d^{2} U}{d r^{2}}+\eta_{1} \delta \frac{d u}{d r}-\eta_{1} \delta \frac{1}{r} U+\left(\eta_{2}-\frac{4}{3} \eta_{4}\right) R \frac{d^{2} \varphi_{1}}{d R^{2}}+\left(\eta_{2}-\frac{4}{3} \eta_{4}\right) \frac{d \varphi_{1}}{d R}- \\
& \left(\eta_{2}-\frac{4}{3} \eta_{4}\right) \frac{1}{R} \varphi_{1}-\frac{4}{3} \eta_{4} R \frac{d^{2} \varphi_{2}}{d R^{2}}-\frac{4}{3} \eta_{4} \frac{d \varphi_{2}}{d R}+\frac{4}{3} \eta_{4} \frac{1}{12} \varphi_{2}=-R^{2} \zeta_{1}
\end{aligned}
$$

$\left(\eta_{2}-\frac{4}{3} \eta_{4}\right) \delta R \frac{d^{2} U}{d R^{2}}+\left(\eta_{2}-\frac{4}{3} \eta_{4}\right) \delta \frac{d U}{d R}-\left(\eta_{2}-\frac{4}{3} \eta_{4}\right) \delta \frac{1}{r} U+$

$\left(\eta_{3}-\frac{8}{3} \eta_{5}+\frac{16}{9}\right) R \frac{d^{2} \varphi_{1}}{d R^{2}}+\left(\eta_{3}-\frac{8}{3} \eta_{5}+\frac{16}{9}\right) \frac{d \varphi_{1}}{d R}-\left[\begin{array}{l}\left(\eta_{3}-\frac{8}{3} \eta_{5}+\frac{16}{9}\right) \frac{1}{R}+ \\ \left(\eta_{6}-8 \eta_{7}+16 \eta_{8}\right) \frac{1}{\delta^{2}} R\end{array}\right] \varphi-\left(\frac{8}{3} \eta_{5}-\frac{16}{9}\right)$

$R \frac{d^{2} \varphi_{2}}{d R^{2}}-\left(\frac{8}{3} \eta_{5}-\frac{16}{9}\right) \frac{d \varphi_{2}}{d R}+\left[\left(\frac{8}{3} \eta_{5}-\frac{16}{9}\right) \frac{1}{R}+\left(4 \eta_{7}+16 \eta_{8}\right) \frac{1}{\delta^{2}} R\right] \varphi_{2}-\left(\eta_{6}+4 \eta_{7}\right)$

$\frac{1}{\delta} R \frac{d w}{d R}=-\zeta_{2} R^{2}$

$\eta_{4} \delta R \frac{d^{2} U}{d R^{2}}+\eta_{4} \delta \frac{d U}{d R}-\eta_{4} \delta \frac{1}{R} U\left(\eta_{5}-\frac{4}{3}\right) R \frac{d^{2} \varphi_{1}}{d R^{2}}+\left(\eta_{5}-\frac{4}{3}\right) \frac{d \varphi_{1}}{d R}-$

$\left[\left(\eta_{5}-\frac{4}{3}\right) \frac{1}{R}+\left(3 \eta_{7}-12 \eta_{8}\right) \frac{1}{\delta^{2}} R\right] \varphi_{1}-\frac{4}{3} R \frac{d^{2} \varphi_{2}}{d R^{2}}-\frac{4}{3} \frac{d \varphi_{2}}{d R}$

$+\left(\frac{4}{3} \frac{1}{R}+12 \eta_{8} \frac{1}{\delta^{2}} R\right) \varphi_{2}-3 \eta_{7} \frac{1}{\delta} R \frac{d w}{d R}=-\zeta_{3} R^{2}$

$$
\begin{aligned}
& \left(\eta_{6}-4 \eta_{7}\right) R \frac{d \varphi_{1}}{d R}+\left(\eta_{6}-4 \eta_{7}\right) \varphi_{1}-4 \eta_{7} R \frac{d \varphi_{2}}{d R}- \\
& 4 \eta_{7} \varphi_{2}+\eta_{7} \delta R \frac{d^{2} w}{d R^{2}}+\delta \eta_{6} \frac{d w}{d R}=-Q R
\end{aligned}
$$

Boundary Conditions

Clamped circular plate

At $R=0$

$U=0, \varphi_{1}=0, \varphi_{2}=0, \frac{d w}{d R}=0, Q_{R}=0$

At $\quad R=1$

$U=0, \varphi_{1}=0, \quad \varphi_{2}=0, W=0$

Roller support circular plate

At $R=0$

$U=0, \varphi_{1}=0, \quad \varphi_{2}=0, \frac{d w}{d R}=0$

At $R=a$

$W=0, N_{r}=0, \quad M_{r}=0 P_{r}=0$

\section{IMPLEMENTATION OF GDQ METHOD}

The generalized differential quadrature (DQ) method is adopted to solve the differential equations of the annular plate. The core of the DQ method is that the derivative of a function in a domain $(0 \leq \mathrm{x} \leq \mathrm{L})$ is approximated as a weighted linear summation of a function values at all discrete points in that domain. Thus, DQ method changes the governing differential equations into a set of corresponding simultaneous equations. To demonstrate the DQ method, consider the rth derivative of a function $f(x)$ can be estimated as

$$
\left.\frac{\partial \mathrm{f}^{\mathrm{r}}(\mathrm{x})}{\partial \mathrm{x}^{\mathrm{r}}}\right|_{X_{i}}=\sum_{\mathrm{k}=1}^{\mathrm{n}} \mathrm{D}_{\mathrm{ik}}^{\mathrm{r}} \mathrm{f}\left(\mathrm{x}_{\mathrm{k}}\right)_{\mathrm{i}=1,2, \ldots \ldots, \mathrm{n}}
$$

Where xi are the discrete points in the variable domain, $\mathrm{D}_{\mathrm{ik}}^{\mathrm{r}}$ , and $\mathrm{f}(\mathrm{xk})$ are the weighting coefficient and the function value at the discrete points.Thus, for the first-order derivatives , the weighting coefficients can be calculated as

$$
\mathrm{D}_{\mathrm{ik}}^{(1)}=\frac{\phi\left(\mathrm{x}_{\mathrm{i}}\right)}{\left(\mathrm{x}_{\mathrm{i}}-\mathrm{x}_{\mathrm{k}}\right) \phi\left(\mathrm{x}_{\mathrm{k}}\right)_{\mathrm{i}}, \mathrm{k}=1,2, \ldots \ldots \mathrm{n}, \mathrm{i} \neq \mathrm{k}}
$$


Where $\phi\left(\mathrm{x}_{\mathrm{i}}\right)=\prod_{\mathrm{i}=1}^{\mathrm{n}}\left(\mathrm{x}_{\mathrm{i}}-\mathrm{x}_{\mathrm{k}}\right)_{\mathrm{i}, \mathrm{k}=1,2, \ldots \ldots \ldots, \mathrm{n}, \mathrm{i} \neq \mathrm{k}}$

After that, the domain of the annular plate is divided into $n$ grade points in $r$ direction. Chebyshev polynomial is the best method to evaluate the grid points in the domain of the plate[25 ]:

$$
r_{i}=0.5^{*}\left[1-\cos \left(\frac{i-1}{n-1} \pi\right)\right]_{i=1,2, \ldots \ldots \ldots n}
$$

The governing eqs. (21) can be discretized according to the GDQ method as follows:

$R_{i} \sum_{j=1}^{n} a_{2}(i, j) u_{j}+\eta_{1} \delta \sum_{j=1}^{n} a_{1}(i, j) u_{j}-\eta_{1} \delta \frac{1}{R_{i}} u_{i}+\left(\eta_{2}-\frac{4}{3} \eta_{4}\right) R_{i} \sum_{j=1}^{n} a_{1}(i, j) \varphi_{j}^{1}+$ $\left(\eta_{2}-\frac{4}{3} \eta_{4}\right) \sum_{j=1}^{n} a_{1}(i, j) \varphi_{j}^{1}-\left(\eta_{2}-\frac{4}{3} \eta_{4}\right) \frac{1}{R_{i}} \varphi_{j}^{1}-$

$\left(\frac{4}{3} \eta_{4}\right) R_{i} \sum_{j=1}^{n} a_{2}(i, j) \varphi_{j}^{2}-\frac{4}{3} \eta_{4} \sum_{j=1}^{n} a_{2}(i, j) \varphi_{j}^{2}+\frac{4}{3} \eta_{4} \frac{1}{R_{i}} \varphi_{j}^{2}=-\zeta_{1} R_{i}^{2}$

$\left(\eta_{2}-\frac{4}{3} \eta_{4}\right) \delta R_{i} \sum_{j=1}^{n} a_{2}(i, j) u_{j}+\left(\eta_{2}-\frac{4}{3} \eta_{4}\right) \delta \sum_{j=1}^{n} a_{1}(i, j) u_{j}-\left(\eta_{2}-\frac{4}{3} \eta_{4}\right) \delta \frac{1}{R_{i}} u_{i}+$ $\left(\eta_{3}-\frac{8}{3} \eta_{5}+\frac{16}{9}\right) R_{i} \sum_{j=1}^{n} a_{2}(i, j) \varphi_{j}^{1}+\left(\eta_{3}-\frac{8}{3} \eta_{5}+\frac{16}{9}\right) \sum_{j=1}^{n} a_{1}(i, j) \varphi_{j}^{1}-$

$\left[\left(\eta_{3}-\frac{8}{3} \eta_{5}+\frac{16}{9}\right) \frac{1}{R_{i}}+\left(\eta_{6}-8 \eta_{7}+16 \eta_{8}\right) \frac{1}{\delta^{2}} R_{i}\right] \varphi_{j}^{1}-\left(\frac{4}{3} \eta_{5}-\frac{16}{9}\right) R_{i} \sum_{j=1}^{n} a_{2}(i, j) \varphi_{j}^{2}$

$-\left(\frac{4}{3} \eta_{5}-\frac{16}{9}\right) \sum_{j=1}^{n} a_{1}(i, j) \varphi_{j}^{2}+\left[\left(\frac{4}{3} \eta_{5}-\frac{16}{9}\right) \frac{1}{R_{i}}+\left(4 \eta_{7}-16 \eta_{8}\right) \frac{1}{\delta^{2}} R_{i}\right]$

$\varphi_{j}^{2}-\left(\eta_{6}-4 \eta_{7}\right) \frac{1}{\delta} R_{i} \sum_{j=1}^{n} a_{1}(i, j) w_{j}=-\zeta_{2} R_{i}^{2}$

(23b)

$\eta_{4} \delta R_{i} \sum_{j=1}^{n} a_{2}(i, j) u_{j}+\eta_{4} \delta \sum_{j=1}^{n} a_{1}(i, j) u_{j}-\eta_{4} \delta \frac{1}{R_{i}} u_{i}+\left(\eta_{5}-\frac{4}{3}\right) R_{i} \sum_{j=1}^{n} a_{2}(i, j) \varphi_{j}^{1}+\left(\eta_{5}-\frac{4}{3}\right)$

$\sum_{j=1}^{n} a_{1}(i, j) \varphi_{j}^{1}-\left[\left(\eta_{5}-\frac{4}{3}\right) \frac{1}{R_{i}}+\left(3 \eta_{7}-12 \eta_{8}\right) \frac{1}{\delta^{2}} R_{i}\right] \varphi_{j}^{1}-\frac{4}{3} R_{i} \sum_{j=1}^{n} a_{2}(i, j) \varphi_{j}^{2}-\frac{4}{3} \sum_{j=1}^{n} a_{1}(i, j) \varphi_{j}^{2}$

$+\left(\frac{4}{3} \frac{1}{R_{i}}+12 \eta_{8} \frac{1}{\delta^{2}} R_{i}\right) \varphi_{j}^{2}-3 \eta_{7} \frac{1}{\delta} R_{i} \sum_{j=1}^{n} a_{1}(i, j) w_{j}=-\zeta_{3} R_{i}^{2}$

$(23 c)$ $\left(\eta_{6}-4 \eta_{h}\right) R_{i} \sum_{j=1}^{n} a_{1}(i, j) \phi_{j}+\left(\eta_{6}-4 \eta_{h}\right) \phi_{j}-$

$\left(4 \eta_{\eta}\right) R_{i} \sum_{j=1}^{n} a_{1}(i, j) \varphi_{j}^{2}-4 \eta_{7} \varphi_{j}^{2}+\eta_{6} \delta R_{i} \sum_{j=1}^{n} a_{2}(i, j) w_{j}+\delta \eta_{6} \sum_{j=1}^{n} a_{1}(i, j) w_{j}=-Q R$

The boundry conditions can be disaretized by the DQ on:

Clampled circular plate .

$$
\begin{gathered}
U_{1}=\varphi_{1}^{1}=\varphi_{1}^{2}=0, \sum_{j=1}^{n} a_{1}(i, j) w_{j}=0 \\
U_{n}=\varphi_{n}^{1}=\varphi_{n}^{2}=W_{n}=0_{R=1}(24 \mathrm{~b})
\end{gathered}
$$

Roller support circular plate .

$$
\begin{aligned}
& U_{1}=\varphi_{1}^{1}=\varphi_{1}^{2}=0 \quad, \quad \sum_{j=1}^{n} a_{1}(i, j) w_{j}=0 \quad \text { at } R=0(25 \mathrm{a}) \\
& W_{n}=0 \\
& \quad \text { at } R=1(25 \mathrm{~b})
\end{aligned}
$$

$\eta_{1} \delta \sum_{j=1}^{n} a_{1}(n, j) u_{j}+\left(v \eta_{1} \delta\right) U_{n}+\left(\eta_{2}-\frac{4}{3} \eta_{4}\right) \sum_{j=1}^{n} a_{1}(n, j) \varphi_{j}^{1}+$ $\left(v \eta_{2}-\frac{4}{3} v \eta_{4}\right) \varphi_{n}^{1}-\frac{4}{3} \eta_{4}$

$\sum_{j=1}^{n} a_{1}(n, j) \varphi_{j}^{2}-\frac{4}{3} v \eta_{4} \varphi_{n}^{2}=0$ at $\mathrm{R}=1$ $\eta_{2} \delta \sum_{j=1}^{n} a_{1}(n, j) u_{j}+\left(v \eta_{2} \delta\right) U_{n}+\left(\eta_{3}-\frac{4}{3} \eta_{5}\right) \sum_{j=1}^{n} a_{1}(n, j) \varphi_{j}^{1}+$ $\left(v \eta_{3}-\frac{4}{3} v \eta_{5}\right) \varphi_{n}^{1}-\frac{4}{3} \eta_{5}$ $\sum_{j=1}^{n} a_{1}(n, j) \varphi_{j}^{2}-\frac{4}{3} v \eta_{5} \varphi_{n}^{2}=0 \ldots \ldots \ldots \ldots$ at $\quad \mathrm{R}=1$ 
$\eta_{4} \delta \sum_{j=1}^{n} a_{1}(n, j) u_{j}+\left(v \eta_{4} \delta\right) U_{n}+\left(\eta_{5}-\frac{4}{3}\right) \sum_{j=1}^{n} a_{1}(n, j) \varphi_{j}^{1}+$

$\left(v \eta_{5}-\frac{4}{3} v\right) \varphi_{n}^{1}-\frac{4}{3} \sum_{j=1}^{n} a_{1}(n, j) \varphi_{j}^{2}$

$-\frac{4}{3} v \varphi_{n}^{2}=0 \ldots \ldots \ldots \ldots$ at $\mathrm{R}=1$

$(25 \mathrm{e})$

The congregation of the governing equations and the related boundary conditions lead to a set of simultaneous linear algebraic equations which can be write in matrix form as:

$$
\left[\begin{array}{ll}
\mathrm{k}_{\mathrm{bb}} & \mathrm{k}_{\mathrm{bd}} \\
\mathrm{k}_{\mathrm{db}} & \mathrm{k}_{\mathrm{dd}}
\end{array}\right]\left\{\begin{array}{c}
\left(\Delta_{\mathrm{b}}\right) \\
\left(\Delta_{\mathrm{d}}\right)
\end{array}\right\}=\left\{\begin{array}{l}
(0) \\
(\mathrm{q})
\end{array}\right\}
$$

$$
\mathrm{k}_{\mathrm{bb}} \quad \mathrm{k}_{\mathrm{bd}}
$$

Where and are the stiffness matrices of boundary of the boundary conditions and the size of it are $8 \times 8$ and $8 \times 4 n$ 8 respectively.

$\mathrm{k}_{\mathrm{db}}$ and $\mathrm{k}_{\mathrm{dd}}$ are stiffness matrices of governing equations and have size of $4 n-8 \times 8$ and $4 n-8 \times 4 n-8$ respectively.

The vector $\Delta_{\mathrm{b}}$ contains the displacements corresponds to the boundary points and is eliminated using the static condensation technique. The stiffness matrix eq.26 can be reduced into the form of

$$
\left[-\mathrm{k}_{\mathrm{db}} \mathrm{k}_{\mathrm{bb}}^{-1} \mathrm{k}_{\mathrm{bd}}+\mathrm{k}_{\mathrm{dd}}\right]\left\{\Delta_{\mathrm{d}}\right\}=\{\mathrm{q}\}
$$

From the above equation the vector of domain displacements $\Delta_{\mathrm{d}}$ can be evaluated.

\section{VALIDATION OF THE RESULTS}

In order to examine the accuracy and efficiency of the results of this paper a comparative study made with another study to implement this , an axisymmetric bending of a clamped and roller-support functionally graded circular plate under uniformly distributed load q . metallic volume fraction power law distribution through plate thickness and all material properties are getting from Reddy et al. [3] As shown in thetable 1 . The evaluation of the comparison .Between the presented numerical analysis and Reddy's exact result are illustrated in table2 for dimensionless maximum deflection,

$\frac{64 W D_{c}}{q a^{4}} \quad D_{c}=\frac{E_{c} h^{3}}{12\left(1-v^{2}\right)}$ With Where $\mathrm{h}$ and a are the thickness and radius of the circular plate. The conclusion from these comparisons, an excellent agreement between these results.

Table 1. Mechanical properties of ceramic and metal of circular FGM plate [ 3 ]

\begin{tabular}{l|cc}
\hline material & $\begin{array}{l}\text { Young's } \\
\text { modulus( Gpa) }\end{array}$ & Poison's ratio,v \\
\hline ceramic & 151 & 0.288 \\
metal & 70 & 0.288
\end{tabular}

Table 2.Comparisons of the result got in the present paper to

\begin{tabular}{|c|c|c|c|c|}
\hline \multirow{2}{*}{$\begin{array}{l}\text { Material } \\
\text { constant } \\
\mathrm{P}\end{array}$} & \multicolumn{2}{|l|}{ Reddy [3] } & \multicolumn{2}{|l|}{ Present } \\
\hline & $\begin{array}{l}\text { Clamped } \\
\text { plate }\end{array}$ & $\begin{array}{l}\text { Roller } \\
\text { support } \\
\text { plate }\end{array}$ & $\begin{array}{l}\text { Clamped } \\
\text { plate }\end{array}$ & $\begin{array}{l}\text { Roller } \\
\text { support } \\
\text { plate }\end{array}$ \\
\hline 0 & 2.979 & 10.822 & 2.979 & 10.822 \\
\hline 2 & 1.623 & 5.925 & 1.608 & 5.921 \\
\hline 4 & 1.473 & 5.414 & 1.467 & 5.410 \\
\hline 6 & 1.404 & 5.155 & 1.399 & 5.150 \\
\hline 8 & 1.362 & 4.993 & 1.357 & 4.989 \\
\hline 10 & 1.333 & 4.882 & 1.329 & 4.880 \\
\hline 15 & 1.289 & 4.714 & 1.287 & 4.713 \\
\hline 20 & 1.265 & 4.619 & 1.264 & 4.613 \\
\hline 25 & 1.250 & 4.559 & 1.248 & 4.558 \\
\hline 30 & 1.239 & 4.517 & 1.238 & 4.5165 \\
\hline 35 & 1.231 & 4.486 & 1.230 & 4.486 \\
\hline 40 & 1.225 & 4.463 & 1.229 & 4.462 \\
\hline 50 & 1.216 & 4.429 & 1.216 & 4.429 \\
\hline 100 & 1.199 & 4.359 & 1.1987 & 4.359 \\
\hline
\end{tabular}
the result got by Reddy etal.[3] for maximum dimantion less diflaction of FGM circular plate for different values of $p$.

\section{RESULTS AND DISCUSSION}

In order to demonstrate the bending and stress analysis of FG circular plate numerically by unconstrained third order shear deformation theory via a generalized differential quadrature method, two cases are studied in this study, clamped circular plate and roller support circular disk .

\subsection{Clamped Circular Plate}

In the below, the results are presented in dimensionless form.

Fig (2) shows the dimensionless deflection $\left(\frac{w}{h}\right)$ along the dimensionless radius $(\mathrm{R})$ under bending load with different values of material constant P.As it expected, the deflection of the metallic plate $(p=0)$ higher than that of the FG plate $(p>0)$, because of the FG plate more stiffer than that of the pure metal plate. 


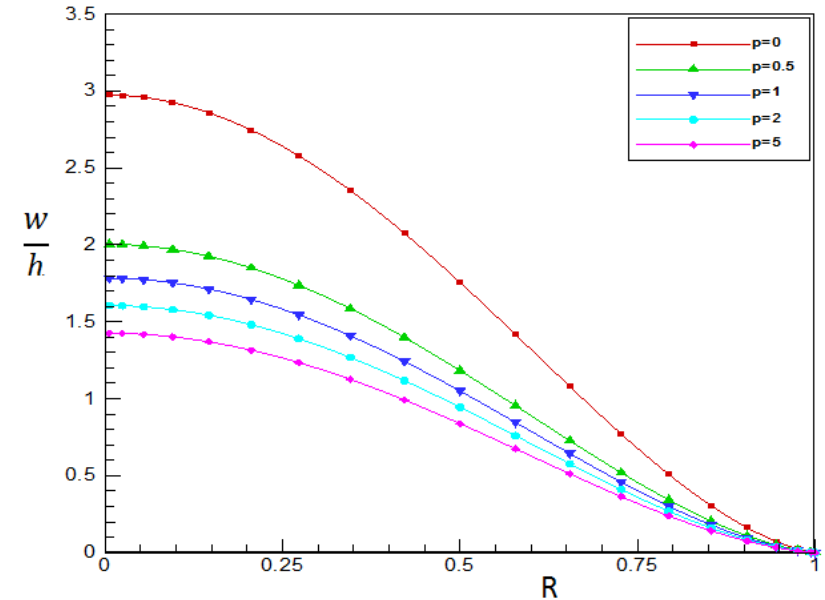

Figure 2:Bending distribution (w/h) for clamped circular platewith $(\mathrm{R})$ for different value of $\mathrm{p}$.

Fig (3) present the variety of the dimensionless shear stress $\left(\tau^{*}=\frac{\tau}{E_{c}}\right)_{\text {through the thickness of the plate with the }}$ dimensionless thickness coordinate variable $\left(\frac{z}{h}\right)$ for different values of material constant $\mathrm{P}$ and plate geometry $\left(\frac{h}{a}\right)$. It is seen that the values of shear stress decrease with increasing in the thickness to the radius ratio $(h / a)$ As well as, it is observable that, there is increasing in the shear stress with the increasing of the material constant $\mathrm{P}$, moreover, it can be seen that the maximum value of shear stress does not occur in the mid plane $\left(\frac{z}{h}=0\right)$, the reason of this case belongs to the nature of non-homogenous of the mechanical properties of the FGMs.

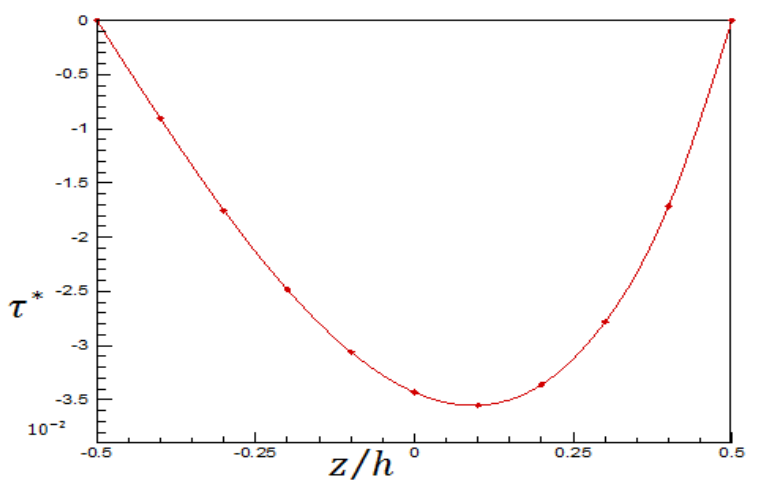

(a)

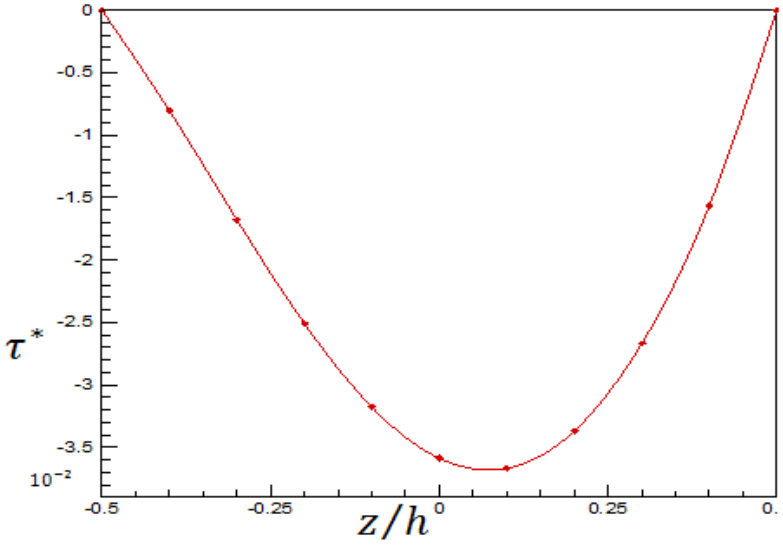

(b)

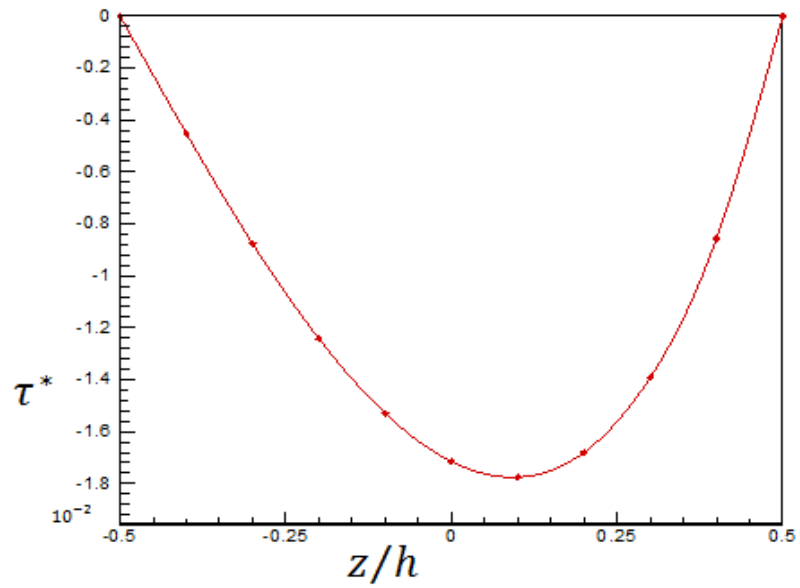

(c)

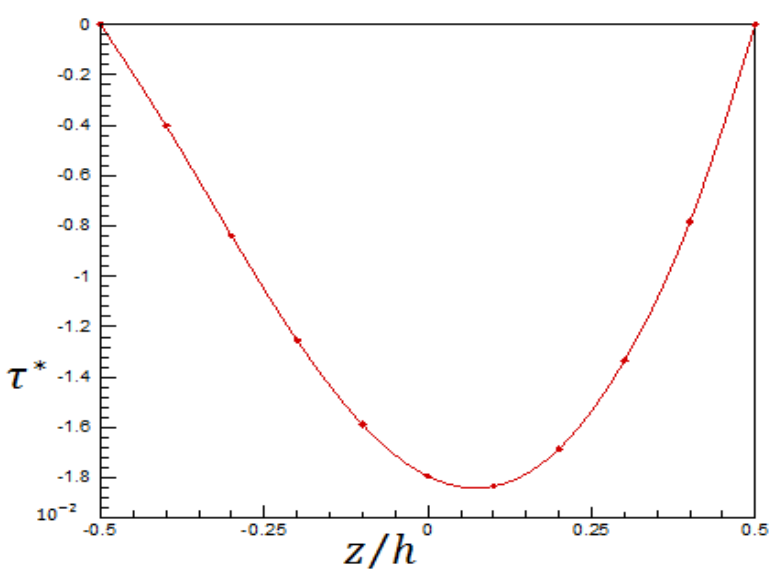

(d)

Figure 3: Shear stress distribution through thikness for clamped circular plate (a) $h / a=0.1, p=0.5$, (b) $\mathrm{h} / \mathrm{a}=0.1, \mathrm{p}=2$, (c) $\mathrm{h} / \mathrm{a}=0.2, \mathrm{p}=0.5$, (d)h/a $=0.2, \mathrm{p}=2$ 


\subsection{Roller Support Circular Disk}

Fig (4) shows the radial stress parameter $\left(\sigma_{r=\frac{\sigma_{r}}{E_{c}}}\right)$ with the body force parameter $(\zeta)$, for different values of the material constantp.it is clear that the redial stress parameter varying linearly with the body force parameter, moreover the radial stress in the FG disk higher than that of pure metallic disk $(P=0)$

Fig (5) illustrates the variation of the radial stress parameter with (R) At $\left(\frac{z}{h}=0.25\right)$ and $\left(\frac{z}{h}=-0.25\right)$.It is clear that under uniform distributed load and body force, the radial stresses for FG plate higher than thatofthe pure metal plate $(P=0)$, because of the FG plate has a higher density.

Fig (6) present the dimensionless deflection $\left(\frac{w}{h}\right)_{\text {with }}(R)$ of the disk under bending load with different values of material constant $p$.it is clear that,the deflection of the pure metal disk $(p=0)$ higher than that of FG plate, because of the rigidty of FG plate.

Fig (7) shows the distribution of shear stress parameter $\left(\tau^{*}\right)$ through the thickness of the plate for different value of thickness to radial variation $\left(\frac{h}{a}\right.$ ) and material constant P .It is clear that the behaviors of the shear stress through the thickness of plate in this case of roller support condition similar to the case of the clamped boundary condition .

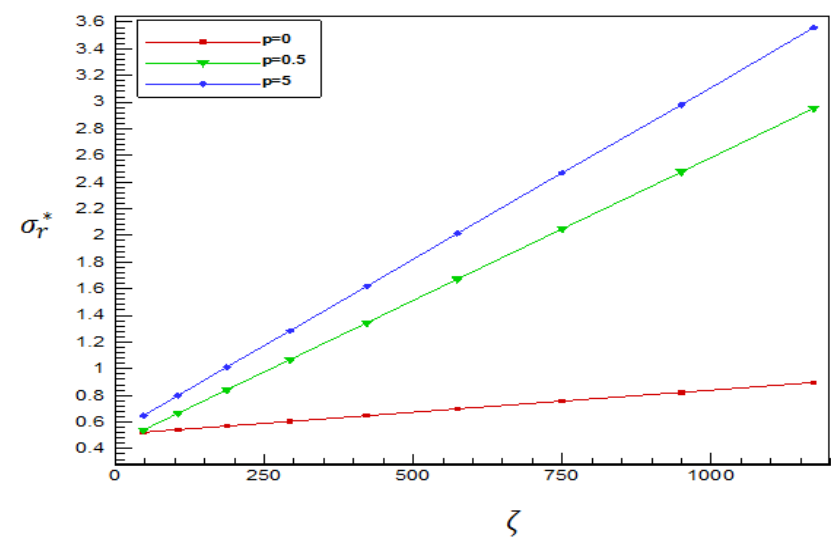

Figure 4: Radial stress $\sigma_{\mathrm{r}}^{*}$ distribution body force $(\zeta)$ for roller support circular disk for different value of $\mathrm{p}$.

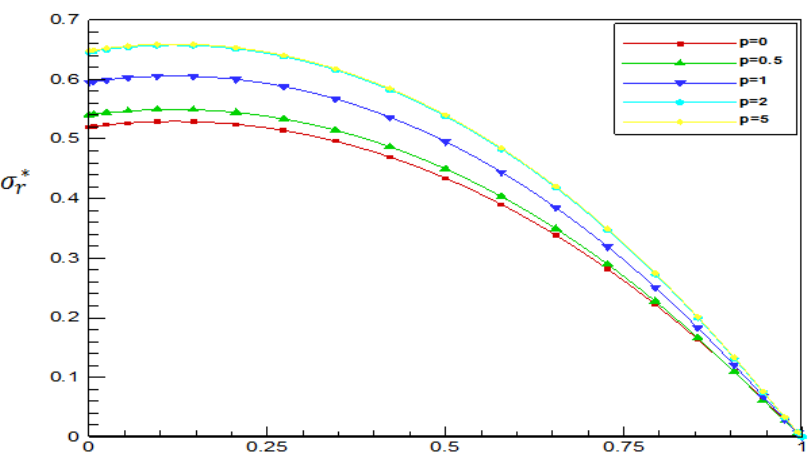

(R)

Figure 5: Radial stress $\sigma_{\mathrm{r}}^{*}$ configralion with $(\mathrm{R})$ at $\frac{\mathrm{Z}}{\mathrm{h}}=0.25$ for roller support circular disk for different value of $\mathrm{p}$.

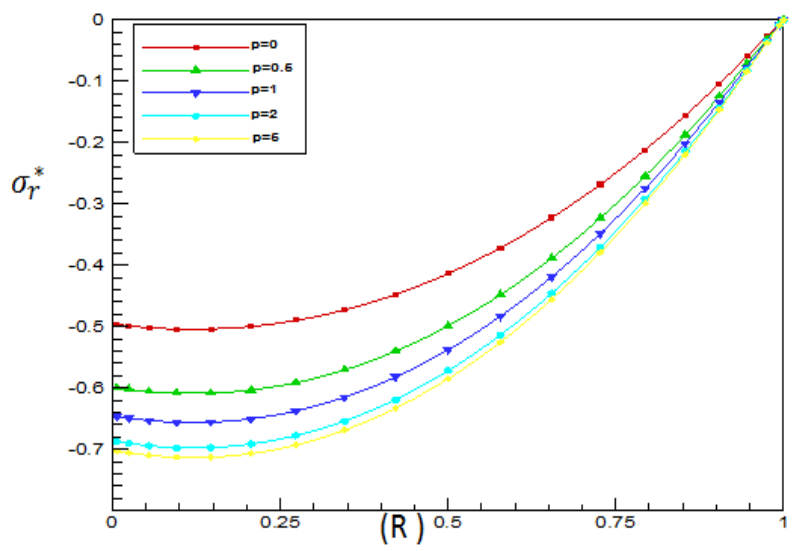

Figure 6: Radial stress $\sigma_{\mathrm{r}}^{*}$ configralion with $(\mathrm{R})$ at $\frac{\mathrm{Z}}{\mathrm{h}}=-0.25$ for roller support circular disk for different value of $\mathrm{p}$.

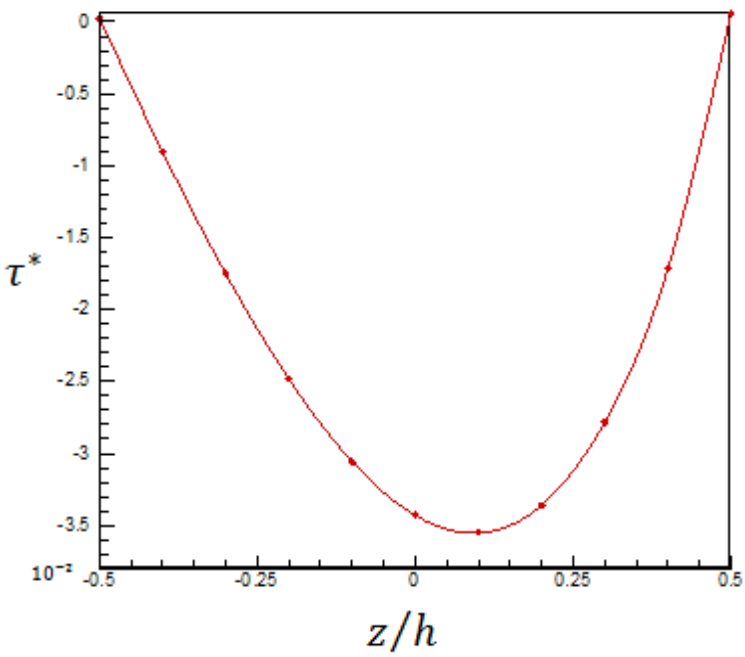

(a) 


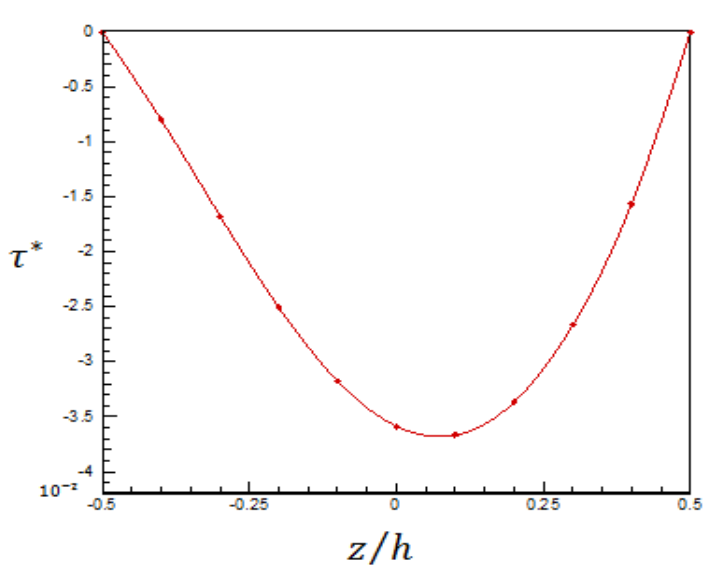

(b)

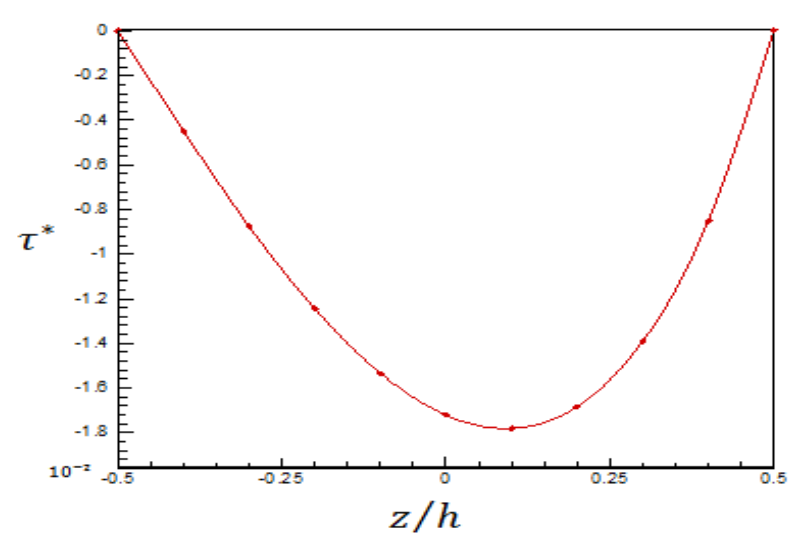

(c)

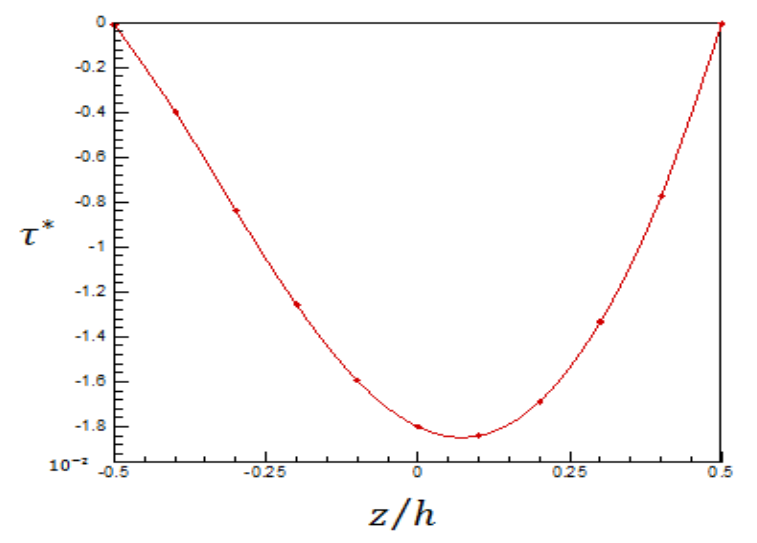

(d)

Figure 7: Shear stress distribution through thikness for roller support circular plate (a) $\mathrm{h} / \mathrm{a}=0.1, \mathrm{p}=0.5$, (b) $\mathrm{h} / \mathrm{a}=0.1, \mathrm{p}=2$, (c) $\mathrm{h} / \mathrm{a}=0.2, \mathrm{p}=0.5$, (d)h/a $=0.2, \mathrm{p}=2$

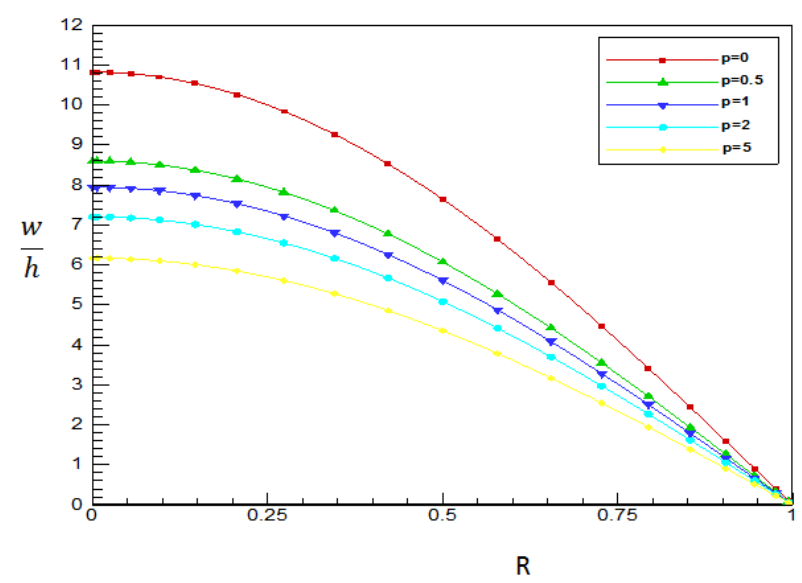

Figure 8: Bending distribution $\left(\frac{w}{h}\right)$ for roller support circular plate with $(\mathrm{R})$ fordifferent value of $\mathrm{p}$.

\section{CONCLUSIONS}

An axismmatric bending and stress analysis of functionally graded circular plate under uniform body force and uniform distributed load by unconstrateed third order shear deformation theory via generalized differential quadrature method (DQM) the numerical solution of the unconstrateed third order shear deformation theory can be applied to different case, of boundary condition, as well as, it can be applied to different loading condition, in contrast to the analytical solution limited to bending load .

\section{REFERENCES}

[1] Koizumi M.1993. the concept of FGM. Ceram Trans Funct Grad Mater;34:310.

[2] Leung Ayt. An unconstrained third order plate theory.Computstruct 1991;40:871-875.

[3] Reddy,J.N., Wang,C.M.,1999. Axisymmetric bending of functionally graded circular and annular plates. Eur.J. Mech, A/solids 18:185-199.

[4] Ma, L.S., Wang,T.S,2004. relationships between ax symmetric bending and buckling solutions of FGM circular plates based on third-order plate theory and classical plate theory. International journal of solids and structures 41:85101.

[5] PrekeshY ,Genepethi M. Axisymmetric flexural vibration and thermoelastic stability of functionally graded material circular plate using finite element method. Compos Part B:Eng 2008, 371:4901-4910.

[6] Dong CI. Three dimensional free vibration analysis of FG annular plates using the chebbyshev-Ritz method. Mater Des 2008,29,1518-1525.

[7] Malekzadeh P, Atashi MM, Karami G. In-plane free vibration of FG circular arches with temperature-dependent 
properties under thermal environment. $\mathbf{J}$ sound vib 2009,326:837-851.

[8] SaidiA.R ,Rasouli A. , Sahraee S. Axisymmetric bending and buckling analysis of FG circular plates using third-order shear deformation theory. Compos struct 2009,89:110-119.

[9] Sahraee,S., Saidi,A. R. Ax symmetric bending analysis of thick functionally graded circular plates using fourth-order shear deformation theory. European journal of mechanics A/solids 2009, 28 :974-984.

[10] Sepahi O., Forouzan M. R. ,Malekzadeh P. Large deflection analysis of thermo-mechanical loaded annular FGM plates on nonlinear elastic foundation via DQM . composstruct 2010,92:2369-2378.

[11] Li S. , Zhang J , Zhao Y. Nonlinear thermo mechanical post-buckling of circular functionally graded material plate with geometric imperfection. Thin-wall struct 2007;45:528536.

[12] MalekzadehP. ,Shahpari S.A., Ziaee H.R. Threedimensional free vibration of thick functionally graded annular plates in thermal environment. J.of sound and vibration 2010;329:425-442.

[13] Nosier A., Fallah F. Nonlinear analysis of functionally graded circular plates under asymmetric transverse loading .int J non linMech 2009;44:928-942.

[14] SburlatiR ,Bandella L. Three-dimensional elastic solutions for FG circular plates. Eur $\mathrm{J}$ Mech A/solids 2011;30:219-235.

[15] GalmekaneEM ,Kedkhodeyan M. Axisymmetric nonlinear bending analysis of annular functionally graded plate using third-order shear deformation theory. Compos Struct 2011;93:973-982.

[16] Hosseini-Hashemish, Fedaee M, Es'haghi M. An exact closed form solution for free vibration of circular and annular moderately thick functionally graded plates based on firstorder shear deformation theory.Int J Mechsci 2010;52:10251035.

[17] NieGJ ,Zhong Z. Frequency analysis of multi-directional functionally graded annular plates by using state space differential quadrature method based on three-dimensional theory of elasticity. Appl Math Model 2010;34:608-616.

[18] Yun W, Rongqiao, Haojiang D. axisymmetric bending of FG circular plates under arbitrary transverse loads. Compos Struct 2010;92:1683-1693.

[19] Ebrahimi E, Rastgoo A, Atai AA. Free vibration of moderately thick shear deformable annular functionally graded plate by using Mindlin's plate theory.Eur J mech A /Solids 2009;28:962-973.

[20] HosseiniSh, Azimzadeh M, Rokni H. A 3-D Ritz solution for free vibration of circular/annular functionally graded plates integrated with piezoelectric layers. Int $\mathbf{J}$ of eng science 2010;48:1971-1984.

[21] L.S Ma , T.J. Wang. Nonlinear bending and postbuckling of functionally graded circular plate under mechanical and thermal loadings. Solids and Struct 2003; 40:3311-3330.
[22] Mehdi Bayat, M. Saleem, B.B.Sari, A.M.S Hamuda. Thermoelastic analysis of functionally graded rotating disk with small and large deflection. Thin-WalStruct 2007; 45:677691.

[23] Erasmo Viola, Luigi Rossetti, Nicholas Fantuzzi. Numerical investigation of functionally graded cylindrical shells and panels using the generalized unconstrained third order theory coupled with the stress recovery. Comp struct $2012 ; 94: 3736-3758$.

[24] Erasmo Viola, Francesco Tornaben , Nicholas Fantuzzi.General higher-order shear deformation theories for the free vibration analysis of completely doubly-curved laminated shells and panels. Comp struct2013 ; 95:639-666.

[25] ShuC.Differential quadrature and its application in engineering.Lonon;Springer, 2000.

\section{BIOGRAPHIES}

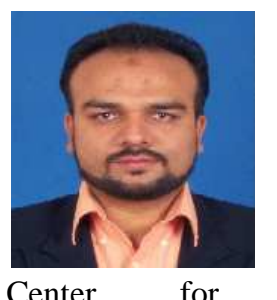

F. Tarlochanwas born in Malaysia. He obtained his Bachelors in Mechanical Engineering and Masters in Biomedical Engineering from Purdue University, USA. His PhD was from Universiti Putra Malaysia. He is currently an Associate Professor at UNITEN and heads the Center for Innovation and Design.Email: faristarlochan@gmail.com

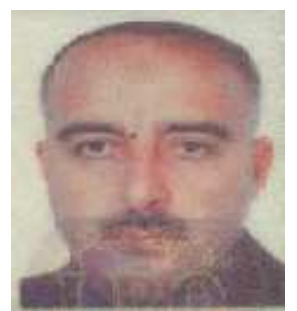

Hamad M. Hwas born in Iraq $\mathrm{He}$ obtained his Bachelors in Mechanical Engineering and Masters in Engineering from IRAQ. He is currently pursuing his $\mathrm{PhD}$ at UNITEN in the field of applied mechanics. 